\title{
HETEROCARYOSIS IN WILD PENICILLIUM
}

\author{
J. L. JINKS \\ Agricultural Research Council's Unit of Biometrical Genetics, \\ Department of Genetics, University of Birmingham
}

Received 5.v.5 I

HETERocaryosis is the association in all the cells of a lineage of nuclei of unlike genetical constitution. These nuclei are presumably haploid and do not normally fuse, although they may do so prior to sexual reproduction, as in Aspergillus nidulans where sexual and heterocaryotic systems co-exist (Pontecorvo, 1946). The work described so far in this field has dealt with heterocaryons derived from two homocaryons that differ by induced biochemical mutations, which in many cases are lethal in the homocaryotic state on a minimal medium (Beadle and Coonradt, I944; Pontecorvo and Gemmell, 1944 $a$ and 1944 $b$; Sansome, I946). These studies on artificial heterocaryons have led to much progress in the investigation of gene action and biochemical syntheses. They have also suggested that heterocaryons may have an importance in nature, but unless the "dual phenomenon" (Hansen, 1938), whose genetical and biological significance has not yet been made clear, is to be regarded as ascribable to heterocaryosis, we have had no evidence so far of the occurrence or operation of the system in wild fungi. The present investigations were undertaken with a view to supplying such evidence.

A recent claim has been made by Papazian (1950) to have produced heterocaryons from pairs of wild homocaryons of Schizophyllum commune. His claim is not, however, borne out by his observations. Twenty single hyphæ were taken from the so-called heterocaryon, but in no case was it possible to show the presence of both original homocaryons in the growth from any one hypha, although the tests for the homocaryons were quite adequate.

\section{ORIGIN OF MATERIAL}

Sixteen examples of the Asymmetrica group of the genus Penicillium were obtained from air, soil and various infected materials, and maintained on a minimal medium, Czapek agar, of following composition :-

\begin{tabular}{|c|c|c|c|c|c|c|}
\hline $\begin{array}{l}\mathrm{NaNO}_{3} \\
\mathrm{KCl} \\
\mathrm{MgSO}_{4} \mathrm{H}_{2} \mathrm{O} \\
\mathrm{FeSO}_{4} \mathrm{H}_{2} \mathrm{O}\end{array}$ & $\begin{array}{l}\dot{ } \\
\dot{\cdot}\end{array}$ & $\begin{array}{l}\dot{5} \\
\dot{5}\end{array}$ & - & $\begin{array}{ll}2.0 & \text { gms. } \\
0.5 & \text { gms. } \\
0.5 & \text { gms. } \\
0.01 & \text { gms. }\end{array}$ & $\begin{array}{l}\mathrm{KH}_{2} \mathrm{PO}_{4} \\
\text { Sucrose } \\
\text { Agar } \\
\text { Water }\end{array}$ & $\begin{array}{l}\text { I } .0 \mathrm{gm} . \\
30 \cdot 0 \mathrm{gms} \text {. } \\
20 \cdot 0 \mathrm{gms} \text {. } \\
\text { I } 000 \mathrm{c.c} .\end{array}$ \\
\hline
\end{tabular}


at $25^{\circ} \mathrm{C}$. They were propagated by single hyphæ. The sources of the various wild isolates are given, along with relative growth rates and other relevant data, in table $\mathrm{I}$.

TABLE I

The 16 wild isolates of the Asymmetrica group of the genus Penicillium

\begin{tabular}{|c|c|c|c|c|}
\hline No. & $\begin{array}{l}\text { Date of } \\
\text { isolation }\end{array}$ & Source & Results & $\begin{array}{l}\text { Relative growth } \\
\text { rate }\end{array}$ \\
\hline I & 7.11 .50 & atmosphere & Heterocaryon & $70 \cdot 6$ \\
\hline 2 & $7.11 .5^{\circ}$ & atmosphere & & $5^{8 \cdot I}$ \\
\hline 4 & $1 \mathrm{I} .1 \mathrm{I} .50$ & atmosphere & Saltated & $72 \cdot 2$ \\
\hline 5 & 12.11 .50 & $\begin{array}{l}\text { saprophyte on capsule of } \\
\text { Nicotiana rustica }\end{array}$ & Heterocaryon & $100 \cdot 0$ \\
\hline 6 & 23.11 .50 & saprophyte on apple & $\cdots$ & $64 \cdot 7$ \\
\hline 7 & 23.11 .50 & saprophyte on marrow & $\cdots$ & $69 \cdot 7$ \\
\hline 8 & 23.11 .50 & saprophyte on bread & $\ldots$ & $91 \cdot 3$ \\
\hline 9 & 25.11 .50 & atmosphere & Homocaryon & $81 \cdot 3$ \\
\hline Ii & 28.11 .50 & atmosphere & Heterocaryon & $72 \cdot 2$ \\
\hline I 2 & 9.12 .50 & saprophyte on lcmon & $\ldots$ & $8 \mathrm{I} \cdot 3$ \\
\hline 13 & 15.12 .50 & saprophyte on cocoanut & Homocaryon & $8 x \cdot 3$ \\
\hline 14 & 3.1 .51 & saprophyte on apple & $\ldots$ & $69 \cdot 7$ \\
\hline 1.5 & $7 \cdot 2.5 \mathrm{I}$ & soil & $\ldots$ & $8 \mathrm{I} \cdot 3$ \\
\hline $1 \ddot{6}$ & $7 \cdot 2.5 \mathrm{I}$ & $\begin{array}{l}\text { saprophyte on secd of } \\
\text { Nicotiana rustica }\end{array}$ & $\ldots$ & $54^{\circ} 0$ \\
\hline I 7 & 7. $2 \cdot 5^{I}$ & soil & $\ldots$ & $86 \cdot 3$ \\
\hline 18 & $7 \cdot 2.5 \mathrm{I}$ & soil & $\ldots$ & $77 \cdot 2$ \\
\hline
\end{tabular}

The relative growth rates are based on No. 5, which is the most thoroughly investigated line to date. These are used because the absolute growth rates vary with each new sample of agar. Thus No. 5 has given growth rates that vary from 9.67 to $12 \cdot 3 \mathrm{~mm}$. per 2 days increase in diameter of its colonies. This variation necessitates a strict system of controls in all comparative work.

All the lines investigated so far have uninucleate conidia, while none have an ascosporic stage, reproduction thus being entirely asexual. No attempt has been made to classify the lines into species, as this is immaterial for the work undertaken. In any case, with the classification of Penicillia in its present state the specics concept has little significance in this group.

\section{BREAKDOWN OF HETEROCARYON No. 5}

The method employed in the investigation of No. 5 will be described as an example of the general technique. No. 5 was obtained as a saprophyte on a capsule of Nicotiana rustica and isolated as a single hypha. It was single sporcd by streaking a drop of a dilute suspension of conidia on to a plate of filtered minimal medium. After incubating for 18 hours at $25^{\circ} \mathrm{C}$. the germinated spores are visible under a low powcr microscope, viewed from the under-surface of the petri dish, and their individual positions can be marked on the under-surface. 
They can then be dissected out by hand from above. Thirty-one colonies were obtained in this way and were found to fall into two distinct groups, $\mathrm{A}$ and $\mathrm{B}$, distinguished by their growth rates. A grew more quickly than $B$ but not so quickly as parent No. 5. Of the 3 I single spore lines so obtained, 24 were of type $\mathrm{A}$ and 7 of type B. An analysis of variance showed that A and B could be regarded as homogeneous groups, and that they differed significantly from one another in growth rate. Fig. I shows the increase in diameter of the colonies of the single spore lines, together with the original parent, during 6 days' growth.

The ratio of the growth rates of No. $5, \mathrm{~A}$ and B are roo : $62: 36 \cdot 5$ based on area, or 10o : $83: 63$ based on diameter, of their colonies

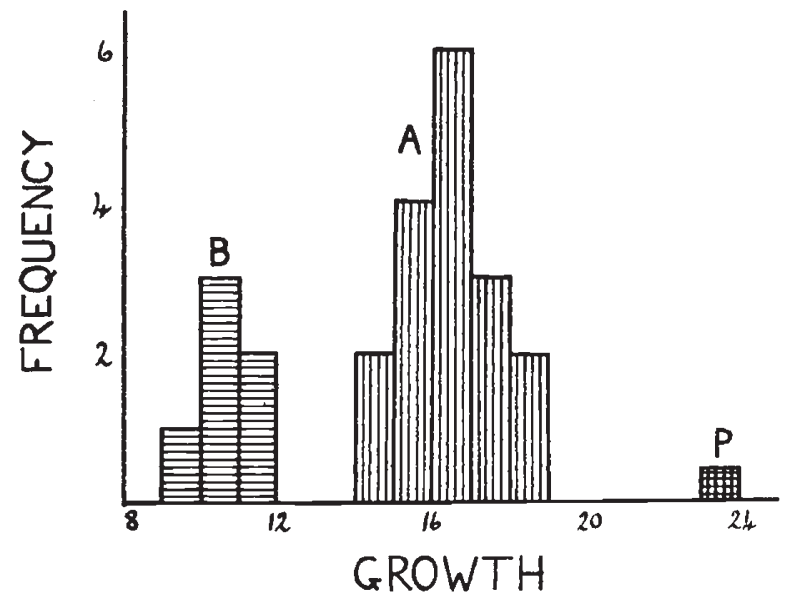

Frg. r. -Distribution histograms of the increase in the diameter, from 2 to 8 days, of the colonies of the single spore lines of No. 5, showing the two homogeneous groups A and $\mathrm{B}$, with the original heterocaryon $\mathrm{P}$ for comparison.

after I2 days' growth. The results from which these ratios were obtained are plotted in fig. 2. Although the absolute growth rates vary with different samples of agar these ratios have shown no significant deviation during four months.

The percentage germination of spores from $\mathrm{A}$ and $\mathrm{B}$ has been investigated at $12^{\circ}, 17^{\circ}$ and $25^{\circ}$ C. No. 5 was also investigated at $12^{\circ} \mathrm{C}$. The results of 3,800 observations are shown in table 2 , and the graph for $12^{\circ} \mathrm{C}$. in fig. 3. Although A grows quicker than $\mathrm{B}$ the reverse relation is shown by their rates of germination. There is a strong suggestion that the conidia from No. 5 are non-autonomous during germination, in that the average rate of germination bears no relation to that expected of a mixture of conidia of type A and $\mathrm{B}$. Spore colour was also found to be non-autonomous for a member of this group. Penicillium notatum, by Pontecorvo and Gemmell (1944a).

As far as morphological characters and appearance of the colonies are concerned, No. 5, A and B are identical. In fact the three lines, 
5, 9 and II which have been studied in detail are indistinguishable except by such physiological characters as rate of growth of colonies and of the germ tubes of conidia.

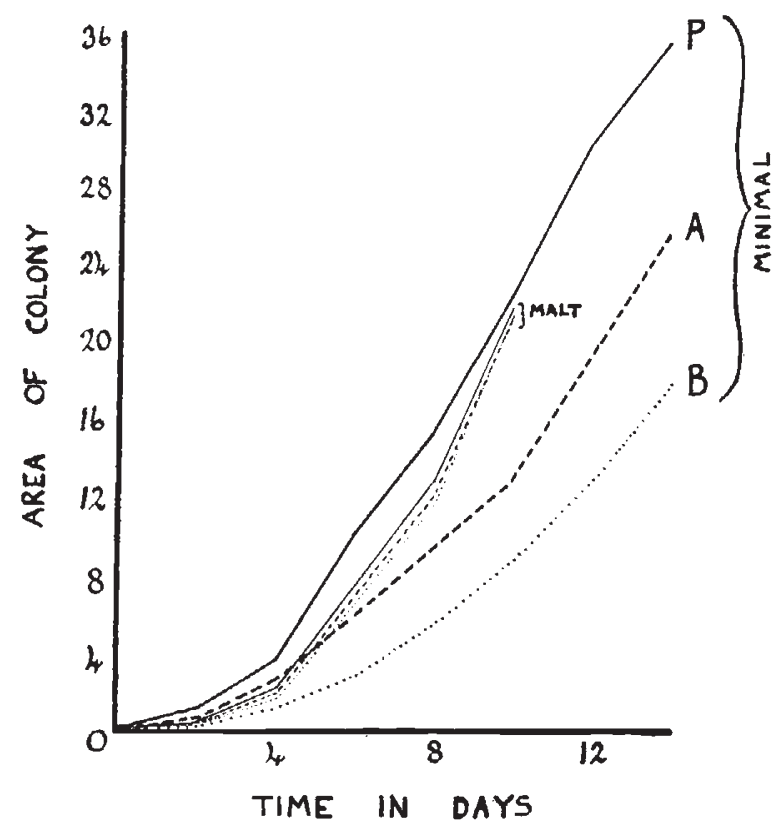

FIG. 2.-Growth curves of the homocaryons $A$ and $B$ and the heterocaryon No. 5 on both a minimal and a 2 per cent. malt extract medium.

TABLE 2

Comparison of the percentage germination of homocaryons $A$ and $B$ at 12,17 and $25^{\circ} \mathrm{C}$. and of heterocaryon $\mathcal{N o} .5$ at $12^{\circ} \mathrm{C}$. at various times

\begin{tabular}{|c|c|c|c|c|c|c|c|}
\hline \multirow{3}{*}{ Time } & \multicolumn{7}{|c|}{ Temperature and type } \\
\hline & \multicolumn{3}{|c|}{$12^{\circ} \mathrm{C}$} & \multicolumn{2}{|c|}{$17^{\circ} \mathrm{C}}$. & \multicolumn{2}{|c|}{$25^{\circ} \mathrm{C}$} \\
\hline & 5 & A & B & A & B & A & B \\
\hline 17 hrs. & I $3 \cdot 8$ & $2 \cdot 0$ & 18.6 & .. & $\cdots$ & 15.4 & $40 \cdot 7$ \\
\hline I $8 \mathrm{hrs}$. & $\cdots$ & $\cdots$ & $\ldots$ & $10 \cdot 0$ & $2 I \cdot 0$ & $\ldots$ & $\ldots$ \\
\hline I9 hrs. & $24 \cdot 6$ & $5^{\cdot I}$ & $\cdots$ & & $\cdots$ & $\cdots$ & \\
\hline $2 \mathrm{I} \mathrm{hrs.}$ & $3^{I} \cdot 4$ & 10.5 & $33 \cdot 5$ & $18 \cdot 3$ & $31 \cdot 6$ & $\cdots$ & $\cdots$ \\
\hline 23 hrs. & $41 \cdot 6$ & $23 \cdot 0$ & $4 \pi \cdot 9$ & $\begin{array}{c}\cdots \\
40 \cdot 0\end{array}$ & $\begin{array}{l}43-2 \\
48 \cdot 0\end{array}$ & $\cdots$ & $\cdots$ \\
\hline 24 & & $\theta$ & 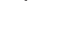 & & & 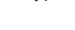 & \\
\hline
\end{tabular}

When grown on a 2 per cent. malt extract agar, which while being richer than the minimal medium is not complete, the growth rates of $5, \mathrm{~A}$ and $\mathrm{B}$ were indistinguishable and differed only slightly from that of 5 on a minimal medium (table 3 and fig. 2). 
It appears, therefore, that we are here dealing with a system akin to the artificial heterocaryons derived from two homocaryons carrying biochemical mutations; the homocaryons growing better on an enriched than on a minimal medium but no better than the heterocaryon on a minimal medium. Such systems differ, however, from

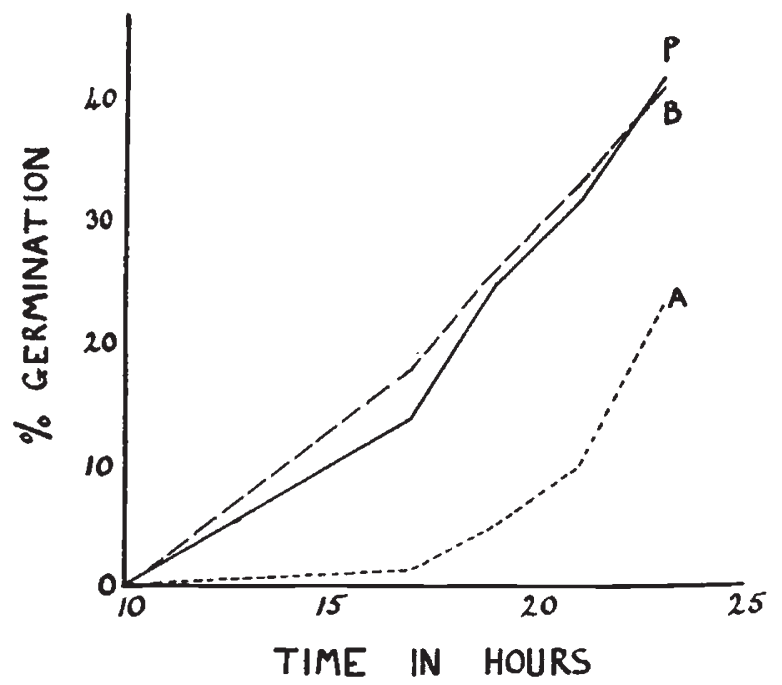

FIG. 3.-Rate of germination of spores from the homocaryons $A$ and $B$ and the heterocaryon No. 5 , at $12^{\circ} \mathrm{C}$. expressed as percentage of germinated spores against time.

TABLE 3

Comparison of the average diameters (in $\mathrm{mm}$.) of the colonies of the homocaryons $A$ and $B$ and heterocaryon No. 5 on 2 per cent. malt extract medium and heterocaryon No. 5 on a minimal medium at 2 day intervals. The last column gives the average increment per 2 days.

\begin{tabular}{|c|c|c|c|c|c|c|c|c|}
\hline Medium & No. & Time & 2 & 4 & 6 & 8 & 10 & Average \\
\hline \multirow{4}{*}{$\begin{array}{c}2 \text { per cent. malt } \\
\text { extract } \\
\text { Minimal }\end{array}$} & A & $2 \cdot 0$ & $9 \cdot 0$ & $19^{\circ} 0$ & $\ldots$ & $39 \cdot 0$ & 53.0 & $10 \cdot 2$ \\
\hline & B & $2 \cdot O$ & $7 \cdot 0$ & $17 \cdot 0$ & $\ldots$ & $3^{8 \cdot 0}$ & $5^{2} \cdot 0$ & $10 \cdot 2$ \\
\hline & 5 & $2 \cdot 0$ & 10.0 & 19.5 & $30 \cdot 0$ & $40 \cdot 0$ & $53^{\circ} 0$ & $10 \cdot 2$ \\
\hline & 5 & $2 \cdot O$ & $12 \cdot 0$ & $22 \cdot 0$ & $36 \cdot 0$ & 45.0 & 53.5 & $10 \cdot 3$ \\
\hline
\end{tabular}

the wild heterocaryon described, in that the homocaryons derived from the wild, while growing better on an enriched medium, are capable of growing satisfactorily, albeit at a slower rate, on a minimal medium. The shortcomings of the wild nuclei are thus less drastic than the biochemical mutations, which are of course lethal on a minimal medium. Indeed, whatever the nature of their inheritance, variants of the kind we find in the wild would not be recovered as laboratory mutants by the standard technique, for in this technique only those induced biochemical variants, which fail to grow at all on a minimal medium are tested further. 


\section{RECONSTITUTION OF THE HETEROCARYON}

The first twelve single spore lines to be isolated, consisting of 9 type $\mathrm{A}$ and 3 type $\mathrm{B}$, were cross tested two at a time for heterocaryon production. Spores of each of the pair of lines to be tested were inoculated on to the same point in the agar. After 6-8 days' growth single hyphæ were taken from the edge of the colony and transferred to new agar plates. Their growth rates were then measured. The removal of single hyphæ was facilitated by the use of plain agar and by the removal of blocks of agar from the path of the colony, so as to promote a sparser growth.

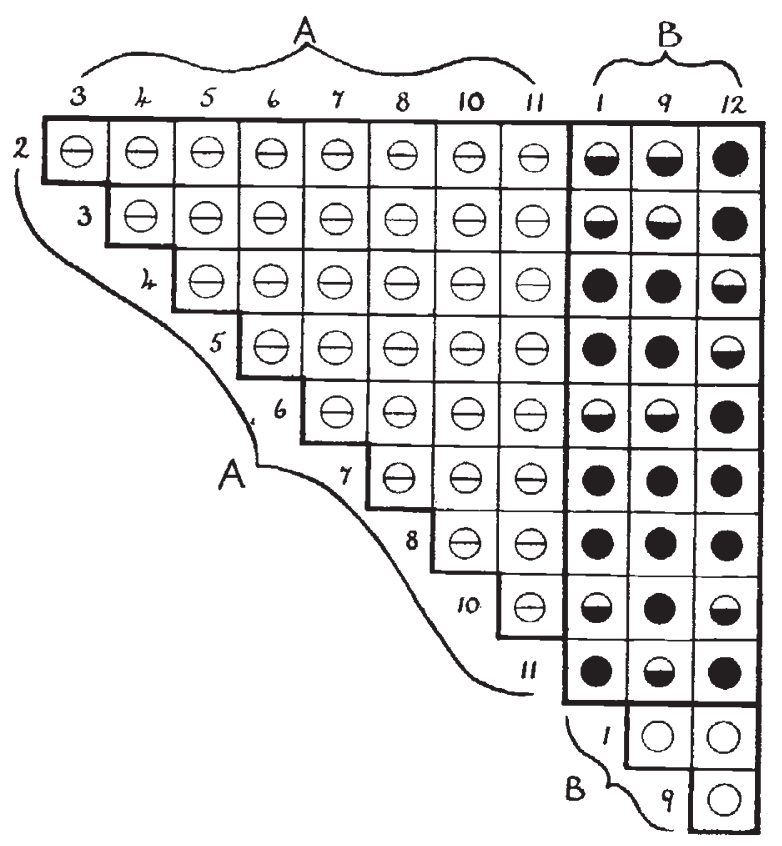

Fig. 4.-Diagram to illustrate the results of the attempts to reconstitute heterocaryon No. 5 from the homocaryons $A$ and B. $\Theta$ denotes combinations giving type A growth rates, $O$ type $B$, heterocaryon growth rate and 0 combinations that gave type $A$ growth rate on first testing but eventually gave the heterocaryon type growth after retesting.

For convenience the experiment, which involves 66 cross tests, was divided into three parts so that each part contained all three types of combinations, i.e. $\mathrm{B}+\mathrm{B}, \mathrm{A}+\mathrm{A}$ and $\mathrm{A}+\mathrm{B}$. In part two, however, the $B+B$ single hyphæ failed to grow. A fourth set of tests was subsequently undertaken to complete the observations. This comprised retests of all $\mathrm{A}+\mathrm{B}$ combinations which failed to produce heterocaryons and also of the cases where the single hyphæ failed to grow in the earlier tests. The results are summarised in fig. 4. All the $\mathrm{A}+\mathrm{A}$ combinations grew at the same rate as type $\mathrm{A}$, and $\mathrm{B}+\mathrm{B}$ combinations similarly gave only type $\mathrm{B}$ growth rate. In 19 
out of the 27 cases of the combination $A+B$, the growth rate was that of the parent heterocaryon. The remaining 8 cases gave the type A growth rate. Heterocaryotic growth was ultimately obtained from these $8 \mathrm{~A}+\mathrm{B}$ combinations after retesting, although as many as six attempts were necessary in some cases before this was achieved.
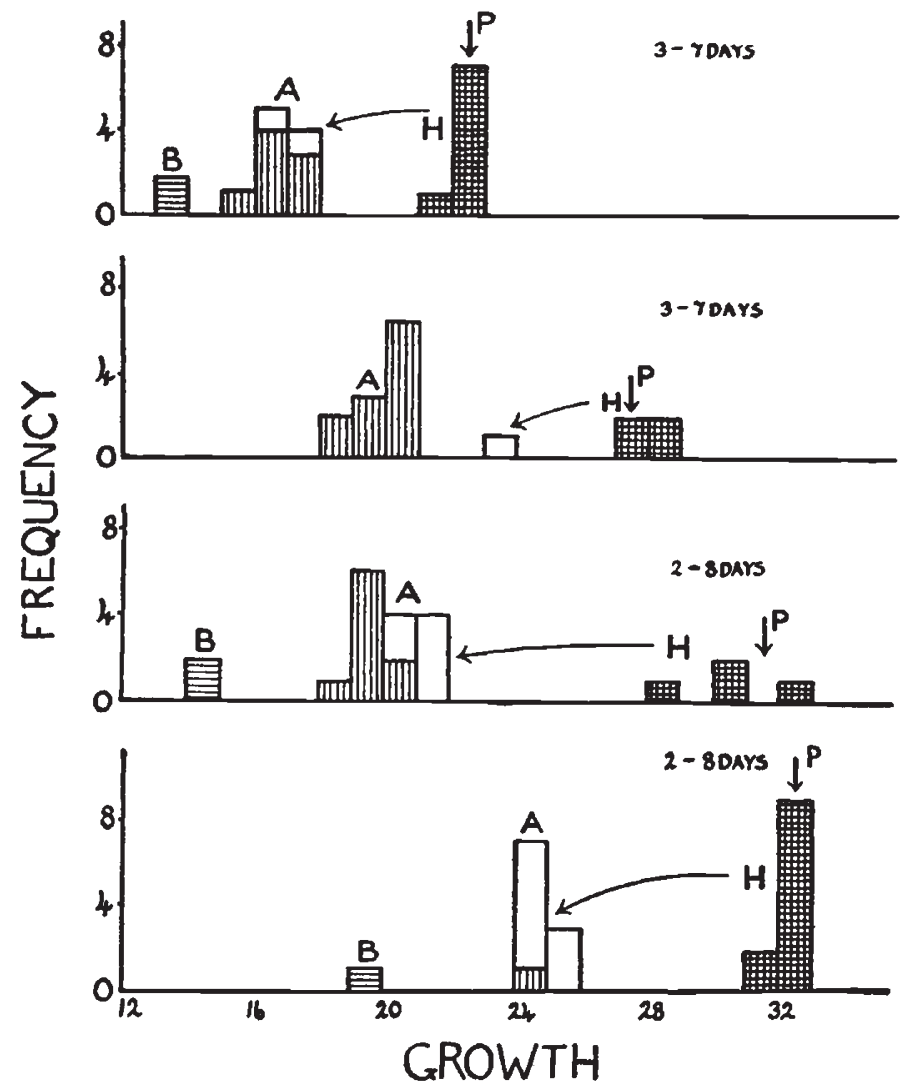

Fig. 5.-Distribution histograms of the increase in diameter of the various combinations of the homocaryon lines, during the time specified. A denotes $\mathrm{A}+\mathrm{A}$ combinations, $B$ denotes $\mathbf{B}+\mathbf{B}$ combinations and $\mathbf{H}$ denotes $\mathbf{A}+\mathbf{B}$ combinations. The unhatched columns with the arrow from $\mathrm{H}$ are the $\mathrm{A}+\mathrm{B}$ combinations that failed to form heterocaryons. $\mathbf{P}$ denotes the increase in diameter of heterocaryon No. 5 under the same conditions.

In all, 57 per cent. of cases where spores of A and B were inoculated together gave heterocaryotic growth.

The actual growth rates of these various combinations are shown as histograms in fig. 5, which is the frequency distribution among the cross tests of the various growth rates observed. Thus in the first set of tests, two of the cultures of combination $B+B$ gave growth between $14^{-15} \mathrm{~mm}$. in diameter while one of type $A+A$ gave a growth of 15-16 mm. in diameter and so on. Reconstituted heterocaryons $\mathrm{H}$ and the original heterocaryon $\mathrm{P}$, caught from the wild, 
had almost identical growth rates in any one set of tests, although the absolute growth rates vary from occasion to occasion, presumably with the medium. The unhatched histograms with the arrow from $\mathrm{H}$ are the growth of colonies of $\mathrm{A}+\mathrm{B}$ combinations that failed to give heterocaryons, giving instead $\mathrm{A}$ growth as might be expected. The histograms marked $A$ and $B$ are the growths of $A+A$ and $B+B$ combinations respectively.

\section{BREAKDOWN OF RECONSTITUTED HETEROCARYONS}

Three of the reconstituted heterocaryons were taken at random and single spored in the same way as was the original heterocaryon. All yielded both types $A$ and $B$ in the same proportions, within sampling error, as did the original heterocaryon, viz. $20 \mathrm{~A}$ to ${ }_{7} \mathrm{~B}$ as compared with $24 \mathrm{~A}$ to $7 \mathrm{~B}$ from the original. As checks, one type $A+A$ and one $B+B$ combination were single spored, and twelve lines grown of each. The first gave all type $A$ and the second all type $B$ growths. With twelve single spore growths and a nuclear ratio of roughly $3 \mathrm{~A}: \mathrm{I} \mathrm{B}$ in the heterocaryons, there was less than one chance in twenty of missing the rarer type of single spore growth had it been present.

Thus the wild parent is a heterocaryon with two genetically distinct types of nucleus, which jointly give a higher growth rate than cither alone.

\section{INVESTIGATION OF No. II}

No. I I, which was obtained from the atmosphere as a single point infection on an exposed plate, has been investigated in the same way as No. 5. On first single sporing only two of the twelve single spores plated gave measurable colonies (type $\mathrm{G}$ ), the other ten produced microscopic growths (type D) that ceased, presumably, when all the food material in the spore was exhausted. Removal of these minute growths to ro per cent. malt extract resulted in little further growth and they finally died. The culture that was single spored was transferred to a new plate, and the transferred colony, on a further single sporing, gave 8 normal colonies, type $\mathrm{C}$, and 2 type $\mathrm{D}$, which even though plated directly on to malt extract, again proved to be inviable. The parent culture was transferred to a new plate once again, in the normal course of maintaining the wild lines. On single sporing for the third time using this new colony, no indication of type $\mathrm{D}$ could be found, all the single spore lines being of type $C$. Type $G$ had a growth rate indistinguishable from that of parent No. I I.

On examining previous data on No. $\mathrm{I} I$ it was found that when first isolated its growth rate was only $3.25 \mathrm{~mm}$. per 2 days, but that after I plate generation, i.e. two weeks, it had risen to $8 \cdot 0 \mathrm{~mm}$. per 2 days. The change could not be due to variations in the agar as all other lines remained constant in growth rate during this period. This suggests that No. I I may have been similar in constitution to 
certain synthetic heterocaryons described by Pontecorvo as being made from two homocaryons one with a wild type growth rate and the other carrying a biochemical mutation that was lethal on a minimal medium. He found that such heterocaryons were unlikely to have a growth rate higher than the wild homocaryon. This results in what Pontecorvo calls unbalanced heterocaryosis, in that a culture started from the heterocaryon resolves itself into the homocaryons, interspersed with a few strips of heterocaryon, that die out as they get further from the centre of inoculation. Obviously single hyphaeing from the edge of such a colony is more likely to yield the homocaryon with the wild type growth rate than anything else. This presumably has happened to our No. I I.

It is not easy to see why a heterocaryon from the wild should be unbalanced, but two possible explanations suggest themselves. First No. I I was a single point infection of an exposed plate. If in the wild it had existed as a balanced heterocaryon of more than two component nuclei, it is possible that only two of these components might have been present in the cluster of conidia which were caught. A more plausible explanation, however, is that on the organic material, on which the parent of the spores which gave rise to No. I I was presumably saprophytic in the wild, the heterocaryon was balanced. The lethal type of nucleus would presumably be able to grow normally if certain food requirements were satisfied, and so play its part in the heterocaryon. This is supported by the observation noted above that when No. I I was first cultured on a minimal medium it grew very slowly, but that its growth soon increased to the rate found for homocaryon $\mathrm{C}$. When first isolated the lethal type may have constituted a fair proportion of the nuclei of the heterocaryon. These would doubtless give different growth properties on the new, minimal, medium than they did on the natural substrate, so that the heterocaryon might well become unbalanced in its fresh circumstances. The lethal nucleus thus decreased in proportion, and as its alternative increased, the growth rate approached that of the homocaryon $\mathrm{C}$, which was isolated from it. Eventually the lethal nucleus died out entirely and the heterocaryon was, in fact, converted into the homocaryon.

It appears, therefore, that No. I I was a heterocaryon consisting of two genetically distinct types of nucleus, one of which carried a mutation that was lethal on a minimal medium, and doubtless similar in type to those produced artificially by X-rays, etc.

\section{OTHER STRAINS}

No. 4 has given a suggestion of being heterocaryotic in that it has saltated to give two types differing in growth rate, neither equal to the original isolate from the wild. Although other possibilities are open, it can be explained as the spontaneous breakdown of a two-phase heterocaryon. This possibility is being tested. 
Only two of our collection, viz. Nos. 9 and 13 , have proved to be homocaryons after careful examination.

\section{DISCUSSION}

The results described leave no doubt that genetical variation exists in wild Penicillium. They also show that heterocaryons are found in the wild and that these can have an advantage over homocaryons similar to that which hybridity confers on individuals of wild out-breeding sexually reproducing species.

An optimum growth rate associated with a certain ratio of the types of nucleus appears to exist for any particular medium. Thus the reconstituted heterocaryons and the original heterocaryon of No. 5 had the same growth rates, within experimental error, on the same medium. Furthermore, those investigated had the same nuclear ratios.

The nuclear ratios appear to be capable of alteration as a result of environmental changes. The change in growth rate of No. I I together with the decrease in the number of lethal nuclei present in the heterocaryon, which was initiated after only one plate generation, suggests a rapid method of readjustment of a heterocaryon to such changes.

If heterocaryosis has such an adaptive significance in wild fungi, it would be expected that means would exist for the regular dispersal and propagation of the heterocaryons. It is of interest in this connection that, although the spores contain only one nucleus, colonies picked up as single point infections on exposed plates have given evidence of being heterocaryotic, e.g. No. I I and No. 4. This suggests that the spores are held together during dispersal. They would thus maintain the heterocaryon in asexual reproduction on which this fungus now relies entirely, having abandoned the sexual cycle.

\section{SUMMARY}

Evidence has been obtained of the regular occurrence of heterocaryons in wild isolates of the Asymmetrica group of the genus Penicillium.

No. 5, isolated as a hyphal tip from a capsule of Nicotiana rustica, gave two types of colonies, $\mathrm{A}$ and $\mathrm{B}$, on single sporing (the spores being uninucleate). A grew faster than B but not so fast as No. 5 . The rates of germination of spores of No. 5, A and B and their growth on malt have also been investigated. No. 5, A and B are morphologically indistinguishable.

The heterocaryon was resynthesised by growing spores from homocaryons $\mathrm{A}$ and $\mathrm{B}$ together. It had a growth rate equal to No. 5 , and, on single sporing, yielded types $\mathrm{A}$ and $\mathrm{B}$ in the same proportions as No. 5 .

No. I I, obtained as a single point infection of an exposed plate, gave two types of single spore colonies, $G$ and D. $G$ had a growth 
rate equal to that of the parent, while $\mathrm{D}$ gave only a microscopic growth on a minimal medium.

Only two isolates out of 16 have so far proved to be homocaryons.

This investigation was carried out while in receipt of a grant from the Agricultural Research Council. I am greatly indebted to Professor K. Mather, Director of the Unit of Biometrical Genetics, for his help and supervision both in the experiments and in the preparation of this paper. I should also like to express my thanks to Mr L. G. Wigan for his critical reading of the manuscript.

\section{REFERENCES}

BEAdle, G. W., AND COONRADT, v. L. 1944. Heterocaryosis in Neurospora crassa. Genetics, 29, 291.

HANSEN, H. N. I938. The dual phenomenon in imperfect fungi. Mycologia, 30, 442 .

PAPAZIAN, H. P. 1950. Physiology of the incompatability factors in Schizophyllum commune. Bot. Gaz., I12, 143 .

PONTECORvo, G. 1946. Genetic systems based on heterocaryosis. Cold Spr. Harb. Sym. quant. Biol., II, 193.

PONTECORVO, G., AND GEMMELl, A. R. 1944a. Genetic proof of heterocaryosis in Penicillium notatum. Nature, 154,514 .

Pontecorvo, G., AND GeMmell, A. R. 1944b. Colonies of Penicillium notatum and other moulds as models for the study of population genetics. Nature, 154, 532. SANSOME, E. 1946. Heterocaryosis, the mating type factors and sexual reproduction in Neurospora. Bul. Torrey Bot. Cl., 73, 397. 